\title{
Some Improved Estimators of Population Mean Under Systematic Sampling
}

\author{
Shagufta Mehnaz ${ }^{1}$ and Shakeel Ahmed ${ }^{* 2}$ \\ ${ }^{1}$ Department of Statistics, university of Lahore, Sargodha Pakistan \\ ${ }^{2}$ Department of Mathematics and Statistics, Institute of Business Management Karachi Pakistan \\ *Corresponding Author: shakeel.ahmed@iobm.edu.pk
}

\begin{abstract}
Auxiliary information is very important in constructing estimators for the population parameters for increasing the efficiency different sampling schemes. In this paper, we consider the problem of estimation of population mean using information on auxiliary variables in systematic sampling. We derive the expressions for the bias and mean squared error (MSE) of the suggested estimators up to the 1st degree of approximation. Proposed estimators are compared with usual mean estimator in systematic sampling scheme theoretically as well as empirically.
\end{abstract}

Keywords: Bias, Mean squared error, Efficiency, Auxiliary information, Systematic sampling.

\section{Introduction}

Utilizing information about the auxiliary variables that are highly correlated with the variable of interest is a common practice for efficiency improvement in estimation of population parameters. Many authors including Cochran (1977), Singh et al. (2004), Khan et al. (2015), Singh et al. (2011), Suberamani and Kumarpandiyan (2013), Sisodia and Dwivedi (1981), Yan and Tian (2010), Upadhyaya and Singh (1999) and Kadilar and Cingi (2004 and 2006) have worked on estimation of population mean using auxiliary information under simple random sampling.

Systematic sampling is preferred over simple random sampling because of two advantages i.e. (1) its simplicity and (2) it give the assurance that the population will be evenly sampled. There may be a chance in simple random sampling that allows of selecting clusters of units in simple random sampling. Systematic sampling overcomes this deficiency by systematically selecting unit from the population. Keeping these advantages in consideration 
many researchers proposed mean estimators under systematic sampling. Works on estimation of finite population mean systematic sampling can be find in Singh and Singh (1998), Singh et al. (2011), Gupta and Shabbir (2008), Suberamani and Kumarpandiyan (2012), Singh et al. (2012), Bahl and tuteja (1991), Tailor et al. (2013), Khan and Singh (2015), Chaudhary and Kumar (2015), Singh (1967).

Let $Y$ be the study variable and $X$ and $Z$ be the auxiliary variables observed on a finite Population $U=\left\{U_{1}, U_{2} \ldots \ldots \ldots U_{N}\right\}$ of size $N$ units numbered from 1 to $N$. To take a systematic sample of size $n$, we divide the population into k mutually exclusive groups or intervals, where $k=N / n$. Then we select $i^{\text {th }}$ unit from first interval randomly and select $i+k, i+2 k, \ldots ., i+(n-1) k^{\text {th }}$ unit from $2^{\text {nd }}, 3^{\text {rd }}, \ldots k^{\text {th }}$ interval respectively. Let $\left(y_{i j}, x_{i j}, z_{i j}\right)$ for $(i=1,2, \ldots . ., k ; j=1,2, \ldots . ., n)$ be the value of $i^{\text {th }}$ unit from $j^{\text {th }}$ interval on variables $Y, X$ and $Z$ respectively. Some important notations are given by

$$
\begin{aligned}
& \bar{X}=\frac{1}{N} \sum_{i=1}^{n} X_{i j}, \quad \bar{Y}=\frac{1}{N} \sum_{j=1}^{n} y_{i j}, \quad \bar{Z}=\frac{1}{N} \sum_{i=1}^{n} z_{i j}, \quad \bar{x}_{s y}=\bar{x}_{i}=\frac{1}{n} \sum_{i=1}^{n} X_{i j}, \quad \bar{y}_{s y}=\bar{y}_{i}=\frac{1}{n} \sum_{j=1}^{n} y_{i j}, \\
& \bar{z}_{s y}=\bar{z}_{i}=\frac{1}{n} \sum_{i=1}^{n} Z_{i j}, \quad S_{x}=\sqrt{\frac{1}{N-1} \sum_{i=1}^{N}\left(x_{i}-\bar{X}\right)^{2}}, \quad S_{y}=\sqrt{\frac{1}{N-1} \sum_{i=1}^{N}\left(y_{i}-\bar{Y}\right)^{2}}, \quad S_{z}= \\
& \sqrt{\frac{1}{N-1} \sum_{i=1}^{N}\left(z_{i}-\bar{Z}\right)^{2}} S_{x y}=\frac{1}{N-1} \sum_{i=1}^{N}\left(y_{i}-\bar{Y}\right)\left(x_{i}-\bar{X}\right), \quad C_{x}=\frac{S_{x}}{\bar{X}}, \quad C_{y}=\frac{S_{y}}{\bar{Y}}, C_{z}=\frac{S_{z}}{\bar{Z}}, \rho_{y x}=\frac{S_{y x}}{S_{y} S_{x}} \\
& \rho_{y x}=\frac{S_{y x}}{S_{y} S_{x}}, \quad \rho_{x z}=\frac{S_{x z}}{S_{x} S_{z}}, \quad \rho_{y z}=\frac{S_{y z}}{S_{y} S_{z}}, \quad \beta_{1(x)}=\frac{\mu_{3}^{2}}{\mu_{2}^{3}}, \quad \beta_{2(x)}=\frac{\mu_{4}}{\mu_{2}^{2}} \quad \text { and } \quad R=\frac{Y}{X}, \quad \text { where } \\
& \sum_{r}^{N}=\frac{\sum_{i=1}^{N}\left(x_{i}-\bar{X}\right)^{r}}{N-1} .
\end{aligned}
$$

Further, we define $M_{d(X)}$ and $M_{d(Z)}$ as the population median of the auxiliary variables $\mathrm{X}$ and $\mathrm{Z}$ respectively.

We also define following error terms

$$
\varepsilon_{o}=\left(\bar{y}_{s y}-\bar{Y}\right) / \bar{Y} \quad, \varepsilon_{1}=\left(\bar{x}_{s y}-\bar{X}\right) / \bar{X}, \varepsilon_{2}={ }^{\left(\bar{z}_{s y}-\bar{Z}\right) / \bar{Z}},
$$

such that $E\left(\varepsilon_{i}\right)=0$ for $i=0,1,2$ and

$$
\begin{gathered}
E\left(e_{0}^{2}\right)=\lambda p_{y}^{*} C_{y}^{2}, \quad E\left(e_{1}^{2}\right)=\lambda p_{x}^{*} C_{x}^{2}, E\left(e_{2}^{2}\right)=\lambda p_{z}^{*} C_{z}^{2} \\
E\left(e_{0} e_{1}\right)=\lambda k C_{x}^{2} \sqrt{p_{y}^{*} p_{x}^{*}}, E\left(e_{0} e_{2}\right)=\lambda k^{*} C_{z}^{2} \sqrt{p_{y}^{*} p_{z}^{*}}, \quad E\left(e_{1} e_{2}\right)=\lambda k^{* *} C_{z}^{2} \sqrt{p_{x}^{*} p_{z}^{*}}
\end{gathered}
$$


where $\quad \rho^{* *}=\frac{\rho_{y}^{*}}{\rho_{x}^{*}}, \rho_{2}^{* *}=\frac{\rho_{y}^{*}}{\rho_{z}^{*}}, \quad \rho_{1}^{* *}=\frac{\rho_{x}^{*}}{\rho_{z}^{*}}, k=\frac{\rho_{y x} C_{y}}{C_{x}}, k^{*}=\frac{\rho_{y z} C_{y}}{C_{z}}, k^{* *}=\frac{\rho_{x z} C_{x}}{C_{z}}$,

$$
\rho_{y}^{*}=\left\{1+(n-1) \rho_{y}\right\}, \quad \rho_{x}^{*}=\left\{1+(n-1) \rho_{x}\right\}, \quad \rho_{z}^{*}=\left\{1+(n-1) \rho_{z}\right\} \quad \text { and } \lambda=\frac{N-1}{n N} \text {. }
$$

We use $\rho_{x}, \rho_{y}$ and $\rho_{z}$ to denote the correlation among the pair units in a systematic sample for the variable $x, y$ and $z$ respectively.

\section{Extension of some well-known estimators in Systematic sampling}

The simple mean estimator under systematic random sampling is given by

$$
\hat{\bar{Y}}_{0}=\frac{1}{n} \sum_{j=1}^{n} y_{i j}=\bar{y}_{i}(i=1,2,3 . . k) \text {. }
$$

It is easy to prove that it is an unbiased estimator of population mean with variance

$$
V\left(\hat{\bar{Y}}_{0}\right)=\lambda \bar{Y}^{2} \rho_{y}^{*} C_{y}^{2}
$$

For efficiency improvement in estimation of mean in systematic sampling we extend Singh et al. (2004), Upadhyaya and Singh (1999), Kadilar and Cigni (2004), Yan and Tian (2010), Sisodia and Dwivedi (1981), Suberamani and Kumarpandiyan (2013) and Tailor et al. (2013) estimators in systematic sampling

$$
\begin{gathered}
\hat{\bar{Y}}_{g(s y)}=\bar{y}_{s y}\left[\frac{a \bar{X}+b}{a \bar{x}_{s y}+b}\right]^{q}\left[\frac{c \bar{Z}+d}{c \bar{z}_{s y}+d}\right]^{p}, \\
\hat{\bar{Y}}_{1(s y)}=\bar{y}_{s y}\left[\frac{\bar{X}+C_{X}}{\bar{x}_{s y}+C_{X}}\right], \\
\bar{Y}_{2(s y)}=\bar{y}_{s y}\left[\frac{\bar{X}+\beta_{2(s y)}}{\bar{x}_{s y}+\beta_{2(s y)}}\right], \\
\hat{\bar{Y}}_{3(s y)}=\bar{y}_{s y}\left[\frac{\bar{X}+\beta_{1(x)}}{\bar{x}_{s y}+\beta_{1(x)}}\right], \\
\hat{\bar{Y}}_{4(s y)}=\bar{y}_{s y}\left[\frac{\bar{X}+\rho_{y x}}{\bar{x}_{s y}+\rho_{y x}}\right], \\
\hat{\bar{Y}}_{5(s y)}=\bar{y}_{s y}\left[\frac{\bar{X}_{x}+\beta_{2(x)}}{\bar{x}_{s y} C_{x}+\beta_{2(x)}}\right],
\end{gathered}
$$




$$
\begin{aligned}
& \hat{\bar{Y}}_{6(s y)}=\bar{y}_{s y}\left[\frac{\bar{X}+M_{d(x)}}{\bar{x}_{s y}+M_{d(x)}}\right], \\
& \hat{\bar{Y}}_{7(s y)}=\frac{\bar{y}_{s y}+b_{y x}\left(\bar{X}-\bar{x}_{s y}\right)}{\bar{x}_{s y}} \bar{X}, \\
& \hat{\bar{Y}}_{8(s y)}=\frac{\bar{y}_{s y}+b_{y x}\left(\bar{X}-\bar{x}_{s y}\right)}{\left(\bar{x}_{s y}+C_{x}\right)}\left(\bar{X}+C_{x}\right), \\
& \hat{\bar{Y}}_{9(s y)}=\frac{\bar{y}_{s y}+b_{y x}\left(\bar{X}-\bar{x}_{s y}\right)}{\left(\bar{x}_{s y}+\beta_{2(x)}\right)}\left(\bar{X}+\beta_{2(x)}\right), \\
& \hat{\bar{Y}}_{10(s y)}=\frac{\bar{y}_{s y}+b_{y x}\left(\bar{X}-\bar{x}_{s y}\right)}{\left(\bar{x}_{s y} C_{X}+\beta_{2(x)}\right)}\left(\bar{X} C_{X}+\beta_{2(x)}\right) \text {, } \\
& \hat{\bar{Y}}_{11(s y)}=\frac{\bar{y}_{s y}+b_{y x}\left(\bar{X}-\bar{x}_{s y}\right)}{\left(\bar{x}_{s y}+\beta_{1(x)}\right)}\left(\bar{X}+\beta_{1(x)}\right), \\
& \hat{\bar{Y}}_{12(s y)}=\frac{\bar{y}_{s y}+b_{y x}\left(\bar{X}-\bar{x}_{s y}\right)}{\left(\bar{x}_{s y} \beta_{1(x)}+\beta_{2(x)}\right)}\left(\bar{X} \beta_{1(x)}+\beta_{2(x)}\right), \\
& \hat{\bar{Y}}_{13(s y)}=\frac{\bar{y}_{s y}+b_{y x}\left(\bar{X}-\bar{x}_{s y}\right)}{\left(\bar{x}_{s y}+\rho_{y x}\right)}\left(\bar{X}+\rho_{y x}\right) \\
& \hat{\bar{Y}}_{14(s y)}=\frac{\bar{y}_{s y}+b_{y x}\left(\bar{X}-\bar{x}_{s y}\right)}{\left(\bar{x}_{s y} C_{X}+\rho_{y x}\right)}\left(\bar{X} C_{X}+\rho_{y x}\right), \\
& \hat{\bar{Y}}_{15(s y)}=\frac{\bar{y}_{s y}+b_{y x}\left(\bar{X}-\bar{x}_{\rho}\right)}{\left(\bar{x}_{\rho}+C_{X}\right)}\left(\bar{X}_{\rho}+C_{X}\right)
\end{aligned}
$$

and

$$
\hat{\bar{Y}}_{16(s y)}=\frac{\bar{y}_{s y}+b_{y x}\left(\bar{X}-\bar{x}_{s y}\right)}{\left(\bar{x}_{s y}+\beta_{2(x)}\right)}\left(\bar{X}_{\rho}+\beta_{2(x)}\right) .
$$

To incorporate information about two auxiliary variables, Tailor et al. (2013) proposed a ratio-cum-product estimator in systematic sampling which is given by 


$$
\hat{\bar{Y}}_{17(s y)}=\bar{y}_{s y}\left(\frac{\bar{X}}{\bar{x}_{s y}}\right)\left(\frac{\bar{Z}}{\bar{z}_{s y}}\right)
$$

The bias of estimators given in Equations (2.3-17) are approximated up to $1^{\text {st }}$ order and the final expressions are given by

$$
\begin{aligned}
& \operatorname{Bias}\left(\hat{\bar{Y}}_{1(s y)}\right) \cong \bar{Y} \rho_{x}^{*} C_{x}^{2} \lambda\left[\theta_{1}^{2}-k \theta_{2} \sqrt{\rho^{* * *}}\right], \\
& \operatorname{Bias}\left(\hat{\bar{Y}}_{3(s y)}\right) \cong \bar{Y} \rho_{x}^{*} C_{x}^{2} \lambda\left[\theta_{3}^{2}-k \theta_{3} \sqrt{\rho^{* * *}}\right], \\
& \operatorname{Bias}\left(\hat{\bar{Y}}_{4(s y)}\right) \cong \bar{Y} \rho_{x}^{*} C_{x}^{2} \lambda\left[\theta_{4}^{2}-k \theta_{4} \sqrt{\rho^{* *}}\right], \\
& \operatorname{Bias}\left(\hat{\bar{Y}}_{5(s y)}\right) \cong \bar{Y} \rho_{x}^{*} C_{x}^{2} \lambda\left[\theta_{5}^{2}-k \theta_{5} \sqrt{\rho^{* *}}\right] \text {, } \\
& \operatorname{Bias}\left(\hat{\bar{Y}}_{6(s y)}\right)=\bar{Y} \rho_{x}^{*} C_{x}^{2} \lambda\left[\theta_{6}^{2}-k \theta_{6} \sqrt{\rho^{* *}}\right] \text {, } \\
& \operatorname{Bias}\left(\hat{\bar{Y}}_{7(s y)}\right) \cong R_{7} \lambda \rho_{x}^{*} \frac{S_{x}^{2}}{\bar{X}}\left[1+\frac{\bar{X}}{\bar{Y}} b_{y x}-k \sqrt{\rho^{* * *}}\right], \\
& \operatorname{Bias}\left(\hat{\bar{Y}}_{8(s y)}\right) \cong R_{8} \lambda \rho_{x}^{*} \frac{S_{x}^{2}}{\bar{X}}\left[1+\frac{\bar{X}}{\bar{Y}} b_{y x}-k \sqrt{\rho^{* *}}\right], \\
& \operatorname{Bias}\left(\hat{\bar{Y}}_{9(s y)}\right) \cong R_{9} \lambda \rho_{x}^{*} \frac{S_{x}^{2}}{\bar{X}}\left[1+\frac{\bar{X}}{\bar{Y}} b_{y x}-k \sqrt{\rho^{* * *}}\right], \\
& \operatorname{Bias}\left(\hat{\bar{Y}}_{10(s y)}\right)=R_{10} \lambda \rho_{x}^{*} \frac{S_{x}^{2}}{\bar{X}}\left[1+\frac{\bar{X}}{\bar{Y}} b_{y x}-k \sqrt{\rho^{* *}}\right] \text {, } \\
& \operatorname{Bias}\left(\hat{\bar{Y}}_{11(s y)}\right) \cong R_{11} \lambda \rho_{x}^{*} \frac{S_{x}^{2}}{\bar{X}}\left[1+\frac{\bar{X}}{\bar{Y}} b_{y x}-k \sqrt{\rho^{* * *}}\right] \text {, } \\
& \operatorname{Bias}\left(\hat{\bar{Y}}_{12(s y)}\right) \cong R_{12} \lambda \rho_{x}^{*} \frac{S_{x}^{2}}{\bar{X}}\left[1+\frac{\bar{X}}{\bar{Y}} b_{y x}-k \sqrt{\rho^{* * *}}\right] \text {, } \\
& \operatorname{Bias}\left(\hat{\bar{Y}}_{13(s y)}\right) \cong R_{13} \lambda \rho_{x}^{*} \frac{S_{x}^{2}}{\bar{X}}\left[1+\frac{\bar{X}}{\bar{Y}} b_{y x}-k \sqrt{\rho^{* *}}\right] \text {, } \\
& \operatorname{Bias}\left(\hat{\bar{Y}}_{14(s y)}\right) \cong R_{14} \lambda \rho_{x}^{*} \frac{S_{x}^{2}}{\bar{X}}\left[1+\frac{\bar{X}}{\bar{Y}} b_{y x}-k \sqrt{\rho^{* * *}}\right] \text {, }
\end{aligned}
$$




$$
\begin{aligned}
& \operatorname{Bias}\left(\hat{\bar{Y}}_{15(s y)}\right) \cong R_{15} \lambda \rho_{x}^{*} \frac{S_{x}^{2}}{\bar{X}}\left[1+\frac{\bar{X}}{\bar{Y}} b-k \sqrt{\rho^{* *}}\right] \\
& \operatorname{Bias}\left(\hat{\bar{Y}}_{16(s y)}\right) \cong R_{16} \lambda \rho_{x}^{*} \frac{S_{x}^{2}}{\bar{X}}\left[1+\frac{\bar{X}}{\bar{Y}} b_{y x}-k \sqrt{\rho^{* * *}}\right] \text {, } \\
& \operatorname{Bias}\left(\hat{\bar{Y}}_{17(s y)}\right) \cong \bar{Y} \theta\left[\rho_{z}^{*} C_{z}^{2}\left(k^{*} \sqrt{\rho_{2}^{* * *}}-k^{* * *} \sqrt{\rho_{1}^{* * *}}\right)+\rho_{x}^{*} C_{x}^{2}\left(1-k \sqrt{\rho^{* * *}}\right)\right] .
\end{aligned}
$$

The Mean Squared Errors of different estimators are approximated up to terms containing square of errors

$$
\begin{gathered}
\operatorname{MSE}\left(\hat{\bar{Y}}_{1(s y)}\right) \cong \bar{Y}^{2} \rho_{x}^{*} \lambda\left[\rho^{* * *} C_{y}^{2}+\theta_{1}^{2} C_{x}^{2}-2 C_{x}^{2} k \theta_{1} \sqrt{\rho^{* * *}}\right], \\
\operatorname{MSE}\left(\hat{\bar{Y}}_{2(s y)}\right) \cong \rho_{x}^{*} \lambda \bar{Y}^{2}\left[\rho^{* * *} C_{y}^{2}+\theta_{2}^{2} C_{x}^{2}-2 C_{x}^{2} k \theta_{2} \sqrt{\rho^{* * *}}\right], \\
\operatorname{MSE}\left(\hat{\bar{Y}}_{3(s y)}\right) \cong \bar{Y}^{2} \rho_{x}^{*} \lambda\left[\rho^{* * *} C_{y}^{2}+\theta_{3}^{2} C_{x}^{2}-2 C_{x}^{2} k \theta_{3} \sqrt{\rho^{* * *}}\right], \\
\operatorname{MSE}\left(\hat{\bar{Y}}_{4(s y)}\right) \cong \bar{Y}^{2} \rho_{x}^{*} \lambda\left[\rho^{* * *} C_{y}^{2}+\theta_{4}^{2} C_{x}^{2}-2 C_{x}^{2} k \theta_{4} \sqrt{\rho^{* * *}}\right], \\
\operatorname{MSE}\left(\hat{\bar{Y}}_{5(s y)}\right) \cong \bar{Y}^{2} \rho_{x}^{*} \lambda\left[\rho^{* * *} C_{y}^{2}+\theta_{5}^{2} C_{x}^{2}-2 C_{x}^{2} k \theta_{5} \sqrt{\rho^{* * *}}\right], \\
\operatorname{MSE}\left(\hat{\bar{Y}}_{6(s y)}\right) \cong \bar{Y}^{2} \rho_{x}^{*} \lambda\left[\rho^{* *} C_{y}^{2}+\theta_{6}^{2} C_{x}^{2}-2 C_{x}^{2} k \theta_{6} \sqrt{\rho^{* * *}}\right], \\
\operatorname{MSE}\left(\hat{\bar{Y}}_{7(s y)}\right) \cong \rho_{x}^{*} \lambda\left[\rho^{* * *} S_{y}^{2}+R_{7}^{2} S_{x}^{2}+\frac{S_{y x}^{2}}{S_{X}^{2}}+2 R S_{y x}-2 R_{7}^{2} S_{x}^{2} k \sqrt{\rho^{* * *}}-2 R_{7} S_{y x} k \sqrt{\rho^{* * *}}\right], \\
\operatorname{MSE}\left(\hat{\bar{Y}}_{8(s y y)}\right) \cong \rho_{x}^{*} \lambda\left[\rho^{* * *} S_{y}^{2}+R_{8}^{2} S_{x}^{2}+\frac{S_{y x}^{2}}{S_{X}^{2}}+2 R_{8} S_{y x}-2 R_{8}^{2} S_{x}^{2} k \sqrt{\rho^{* * *}}-2 R_{8} S_{y x} k \sqrt{\rho^{* *}}\right], \\
\operatorname{MSE}\left(\hat{\bar{Y}}_{9(s y)}\right) \cong \rho_{x}^{*} \lambda\left[\rho^{* * *} S_{y}^{2}+R_{9}^{2} S_{x}^{2}+\frac{S_{y x}^{2}}{S_{X}^{2}}+2 R_{9} S_{y x}-2 R_{9}^{2} S_{x}^{2} k \sqrt{\rho^{* * *}}-2 R_{9} S_{y x} k \sqrt{\rho^{* *}}\right], \\
\operatorname{MSE}\left(\hat{\bar{Y}}_{10(s y)}\right) \cong \rho_{x}^{*} \lambda\left[\rho^{* * *} S_{y}^{2}+R_{10}^{2} S_{x}^{2}+\frac{S_{y x}^{2}}{S_{X}^{2}}+2 R_{10} S_{y x}-2 R_{10}^{2} S_{x}^{2} k \sqrt{\rho^{* * *}}-2 R_{10} S_{y x} k \sqrt{\rho^{* * *}}\right],
\end{gathered}
$$




$$
\begin{aligned}
& \operatorname{MSE}\left(\hat{\bar{Y}}_{11(s y)}\right) \cong \rho_{x}^{*} \lambda\left[\rho^{* *} S_{y}^{2}+R_{11}^{2} S_{x}^{2}+\frac{S_{y x}^{2}}{S_{X}^{2}}+2 R_{11} S_{y x}-2 R_{11}^{2} S_{x}^{2} k \sqrt{\rho^{* *}}-2 R_{11} S_{y x} k \sqrt{\rho^{* * *}}\right], \\
& \operatorname{MSE}\left(\hat{\bar{Y}}_{12(s y)}\right)=\rho_{x}^{*} \lambda\left[\rho^{* *} S_{y}^{2}+R_{12}^{2} S_{x}^{2}+\frac{S_{y x}^{2}}{S_{X}^{2}}+2 R_{12} S_{y x}-2 R_{12}^{2} S_{x}^{2} k \sqrt{\rho^{* *}}-2 R_{12} S_{y x} k \sqrt{\rho^{* *}}\right] \text {, } \\
& \operatorname{MSE}\left(\hat{\bar{Y}}_{13(s y)}\right) \cong \rho_{x}^{*} \lambda\left[\rho^{* *} S_{y}^{2}+R_{13}^{2} S_{x}^{2}+\frac{S_{y x}^{2}}{S_{X}^{2}}+2 R_{13} S_{y x}-2 R_{13}^{2} S_{x}^{2} k \sqrt{\rho^{* *}}-2 R_{13} S_{y x} k \sqrt{\rho^{* *}}\right] \text {, } \\
& \operatorname{MSE}\left(\hat{\bar{Y}}_{14(s y)}\right) \cong \rho_{x}^{*} \lambda\left[\rho^{* * *} S_{y}^{2}+R_{14}^{2} S_{x}^{2}+\frac{S_{y x}^{2}}{S_{X}^{2}}+2 R_{14} S_{y x}-2 R_{14}^{2} S_{x}^{2} k \sqrt{\rho^{* * *}}-2 R_{14} S_{y x} k \sqrt{\rho^{* * *}}\right] \text {, } \\
& \operatorname{MSE}\left(\bar{Y}_{15(s y)}\right) \cong \rho_{x}^{*} \lambda\left[\rho^{* *} S_{y}^{2}+R_{15}^{2} S_{x}^{2}+\frac{S_{y x}^{2}}{S_{X}^{2}}+2 R_{15} S_{y x}-2 R_{15}^{2} S_{x}^{2} k \sqrt{\rho^{* * *}}-2 R_{15} S_{y x} k \sqrt{\rho^{* *}}\right], \\
& \operatorname{MSE}\left(\hat{\bar{Y}}_{16(s y)}\right) \cong \rho_{x}^{*} \lambda\left[\rho^{* *} S_{y}^{2}+R_{16}^{2} S_{x}^{2}+\frac{S_{y x}^{2}}{S_{X}^{2}}+2 R_{16} S_{y x}-2 R_{16}^{2} S_{x}^{2} k \sqrt{\rho^{* *}}-2 R_{16} S_{y x} k \sqrt{\rho^{* *}}\right], \\
& \operatorname{MSE}\left(\hat{\bar{Y}}_{17(s y)}\right) \cong \lambda \bar{Y}^{2}\left[\rho_{y}^{*} C_{y}^{2}+\frac{\rho_{x}^{*} C_{x}^{2}}{4}\left(1-2 K \sqrt{\rho^{* *}}\right)+\rho_{z}^{*} C_{z}^{2}\left(1-2 k^{* *} \sqrt{\rho_{1}^{* * *}}\right)+2 k^{*} C_{z}^{2} \sqrt{\rho_{y}^{*} \rho_{z}^{*}}\right] \text {, }
\end{aligned}
$$

\section{Proposed Estimators in Systematic Random Sampling}

On the basis of the fact that median of the auxiliary variable is easy to obtain from the population being studied, we suggest some more efficient estimators in systematic sampling by following Suberamani and Kumarpandiyan (2013) as follows

$$
\begin{aligned}
& \hat{\bar{Y}}_{18 p(s y)}=\bar{y}_{s y}\left[\frac{\bar{X} \beta_{1(x)}+M_{d(x)}}{\bar{x}_{s y} \beta_{1(x)}+M_{d(x)}}\right], \\
& \hat{\bar{Y}}_{19 p(s y)}=\bar{y}_{s y}\left[\frac{\bar{X} M_{d(x)}+\beta_{1(x)}}{\bar{x}_{s y} M_{d(x)}+\beta_{1(x)}}\right],
\end{aligned}
$$




$$
\begin{aligned}
& \hat{\bar{Y}}_{20 p(s y)}=\bar{y}_{s y}\left[\frac{\bar{X} M_{d(x)}+\beta_{2(x)}}{\bar{x}_{s y} M_{d(x)}+\beta_{2(x)}}\right], \\
& \hat{\bar{Y}}_{21 p(s y)}=\frac{\bar{y}_{s y}+b_{y x}\left(\bar{X}-\bar{x}_{s y}\right)}{\left(\bar{x}_{s y}+M_{d(x)}\right)}\left(\bar{X}+M_{d(x)}\right) .
\end{aligned}
$$

Taking inspiration from Tailor et al. (2013), we proposed the following ratio and product type estimator, for using median along with mean two auxiliary variable.

$$
\hat{\bar{Y}}_{22 p(s y)}=\bar{y}_{s y}\left[\frac{\bar{X}+M_{d(x)}}{\bar{x}_{s y}+M_{d(x)}}\right]\left[\frac{\bar{Z}+M_{d(z)}}{\bar{z}_{s y}+M_{d(z)}}\right],
$$

where $M_{d(X)}$ and $M_{d(Z)}$ are the population median of auxiliary variable $X$ and $Z$ respectively

The bias of estimators given in Equations (3.1-5) are given by

$$
\begin{gathered}
\operatorname{Bias}\left(\hat{\bar{Y}}_{18(s y)}\right)=\bar{Y} \rho_{x}^{*} C_{x}^{2} \lambda\left[\theta_{18}^{2}-k \theta_{18} \sqrt{\rho^{* * *}}\right], \\
\operatorname{Bias}\left(\hat{\bar{Y}}_{19(s y)}\right)=\bar{Y} \rho_{x}^{*} C_{x}^{2} \lambda\left[\theta_{19}^{2}-k \theta_{19} \sqrt{\rho^{* *}}\right], \\
\operatorname{Bias}\left(\hat{\bar{Y}}_{20(s y)}\right)=\bar{Y} \rho_{x}^{*} C_{x}^{2} \lambda\left[\theta_{20}^{2}-k \theta_{20} \sqrt{\rho^{* *}}\right], \\
\operatorname{Bias}\left(\hat{\bar{Y}}_{21(s y)}\right)=R_{21} \lambda \rho_{x}^{*} \frac{S_{x}^{2}}{\bar{X}}\left[1+\frac{\bar{X}}{\bar{Y}} b_{y x}-k \sqrt{\rho^{* *}}\right]
\end{gathered}
$$

and

$$
\operatorname{Bias}\left(\hat{\bar{Y}}_{22(s y)}\right)=\bar{Y} \lambda\left[b_{22}^{2} \rho_{y}^{*} C_{y}^{2}+\rho_{x}^{*} C_{x}^{2}\left(a_{22}^{2}-a_{22} k \sqrt{\rho^{* *}}\right)+\rho_{z}^{*} C_{z}^{2}\left(a_{22} b_{22} k^{* *} \sqrt{\rho_{1}^{* *}}-b_{22} k^{*} \sqrt{\rho_{2}^{* *}}\right] .\right.
$$

The Mean Squared Error of proposed estimators given in Equations (3.1-5) are expressed as follow

$$
\begin{gathered}
\operatorname{MSE}\left(\hat{\bar{Y}}_{18(s y)}\right)=\bar{Y}^{2} \rho_{x}^{*} \lambda\left[\rho^{* *} C_{y}^{2}+\theta_{18}^{2} C_{x}^{2}-2 C_{x}^{2} k \theta_{18} \sqrt{\rho^{* *}}\right], \\
\operatorname{MSE}\left(\hat{\bar{Y}}_{19(s y)}\right)=\bar{Y}^{2} \rho_{x}^{*} \lambda\left[\rho^{* *} C_{y}^{2}+\theta_{19}^{2} C_{x}^{2}-2 C_{x}^{2} k \theta_{19} \sqrt{\rho^{* *}}\right], \\
\operatorname{MSE}\left(\hat{\bar{Y}}_{20(s y)}\right)=\bar{Y}^{2} \rho_{x}^{*} \lambda\left[\rho^{* *} C_{y}^{2}+\theta_{20}^{2} C_{x}^{2}-2 C_{x}^{2} k \theta_{20} \sqrt{\rho^{* *}}\right],
\end{gathered}
$$




$$
\operatorname{MSE}\left(\hat{\bar{Y}}_{21(s y)}\right)=\rho_{x}^{*} \lambda\left[\rho^{* *} S_{y}^{2}+R_{21}^{2} S_{x}^{2}+\frac{S_{y x}^{2}}{S_{X}^{2}}+2 R_{21} S_{y x}-2 R_{21}^{2} S_{x}^{2} k \sqrt{\rho^{* *}}-2 R_{21} S_{y x} k \sqrt{\rho^{* *}}\right]
$$

and

$$
\begin{aligned}
& \operatorname{MSE}\left(\hat{\bar{Y}}_{22(s y)}\right)=\bar{Y}^{2} \theta\left[\rho_{y}^{*} C_{y}^{2}+\rho_{x}^{*} C_{x}^{2}\left(a^{2}-2 a_{22} k \sqrt{\rho^{* * *}}\right)+\rho_{z}^{*} C_{z}^{2}\left(b^{2}+2 a_{22} b_{22} k^{* * *} \sqrt{\rho_{1}^{* * *}}-2 b_{22} k^{*} \sqrt{\rho_{2}^{* * *}}\right],\right. \\
& \text { where } \quad \theta_{18}=\frac{\bar{X} \beta_{1(x)}}{\bar{X} \beta_{1(x)}+M_{d(x)}}, \theta_{19}=\frac{\bar{X} M_{d(x)}}{\bar{X} M_{d(x)}+\beta_{1(x)}}, \\
& R_{21}=\left[\frac{\bar{Y}}{\bar{X}+M_{d(x)}}\right], a_{22}=\frac{\bar{X}}{\bar{X}+M_{d(x)}} \text { and } b_{22}=\frac{\bar{Z}}{\bar{Z}+M_{d(x)}} .
\end{aligned}
$$

\section{Numerical Study}

A real life data set is used, to exhibit the performance of the proposed estimators.

\section{Data set: (Source. 1)}

We use District Wise Maize Data of Punjab the province of Pakistan for year 2014, where

$\mathrm{Y}=$ Production of Maize for year 2007-08 (in tons),

$X=$ Area of Maize for year 2007-08 (in hectares sq),

$Z=$ Area of Maize for year 2008-09 (in hectares sq ) .

The Values of population parameters and the constants obtained from the above data are given below:

$$
\begin{gathered}
N=25, \lambda=4.8, n=5, k=1.218752, K^{*}=1.24581, K^{* *}=1.019864, \beta_{1}(x)=0.0018, \\
\beta_{2}(x)=4.2872, \bar{X}=19.192, C_{x}=1.390563, S_{x}^{2}=712.2324, \rho_{x}^{*}=0.205311, \bar{Y}=97.016, \\
C_{y}=1.795732, S_{y}^{2}=30350.79, \rho_{y}^{*}=0.258816, \bar{Z}=18.86, C_{z}=1.360231, S_{z}^{2}=658.1258, \\
\rho_{z}^{*}=0.204505, \rho_{y x}=0.94376, \rho^{* *}=1.260606, S_{y z}=4387.935, M_{d}(x)=4.7, \rho_{y z}=0.94367, \\
\rho_{1}^{* *}=1.003939, S_{x z}=683.0143, M_{d}(z)=5.1, \rho_{x z}=0.99762, \rho_{2}^{* *}=1.265572, S_{y x}=4217.565 .
\end{gathered}
$$

The biases, mean squared errors and percentage relative efficiencies (PREs) of different estimator in systematic sampling are given in Table (4.1). Using the following formula;

$$
\operatorname{PRE}\left(\hat{\bar{Y}}_{a(s y)}, \hat{\bar{Y}}_{0(s y)}\right)=\frac{\operatorname{Var}\left(\hat{\bar{Y}}_{0(s y)}\right)}{\operatorname{MSE}\left(\hat{\bar{Y}}_{a(s y)}\right)} \times 100 \text { for } a=0,1,2, \ldots \ldots . ., 22 .
$$

where $\operatorname{PRE}\left(\hat{\bar{Y}}_{a(s y)}, \hat{\bar{Y}}_{0(s y)}\right)$ denotes the percentage relative efficiency of $a^{\text {th }}$ estimator 
Table 1: The Absolute Biases, MSE'S and PRE'S of Different Estimators under Systematic Random Sampling

\begin{tabular}{|l|c|c|c|}
\hline Estimator & $\left|\operatorname{Bias}\left(\hat{\bar{Y}}_{a}\right)\right|$ & $\operatorname{MSE}\left(\hat{\bar{Y}}_{a}\right)$ & $\operatorname{PRE}\left(\hat{\bar{Y}}_{a}, \hat{\bar{Y}}_{0}\right)$ \\
\hline$\hat{\bar{Y}}_{0(s y)}$ & 0.000 & 37705.26 & 100 \\
\hline$\hat{\bar{Y}}_{1(s y)}$ & 75.1484 & 7529.906 & 500.7401 \\
\hline$\hat{\bar{Y}}_{2(s y)}$ & 68.1141 & 6556.526 & 575.0798 \\
\hline$\hat{\bar{Y}}_{3(s y)}$ & 83.2613 & 8591.218 & 438.8815 \\
\hline$\hat{\bar{Y}}_{4(s y)}$ & 73.1701 & 7214.039 & 522.6651 \\
\hline$\hat{\bar{Y}}_{5(s y)}$ & 68.111 & 6556.178 & 575.1104 \\
\hline$\hat{\bar{Y}}_{6(s y)}$ & 83.9199 & 9848.845 & 382.8394 \\
\hline$\hat{\bar{Y}}_{7(s y)}$ & 157.2131 & 17091.51 & 220.6081 \\
\hline$\hat{\bar{Y}}_{8(s y)}$ & 172.3844 & 22246.38 & 169.4894 \\
\hline$\hat{\bar{Y}}_{9(s y)}$ & 157.1984 & 17098.87 & 220.5132 \\
\hline$\hat{\bar{Y}}_{10(s y)}$ & 157.2025 & 17096.8 & 220.5398 \\
\hline$\hat{\bar{Y}}_{1(s y)}$ & 128.5061 & 26246.71 & 143.6571 \\
\hline$\hat{\bar{Y}}_{12(s y)}$ & 157.2097 & 17093.23 & 220.5859 \\
\hline$\hat{\bar{Y}}_{13(s y)}$ & 149.8445 & 20697.92 & 182.1693 \\
\hline$\hat{\bar{Y}}_{14(s y)}$ & 151.8434 & 19733.13 & 191.0759 \\
\hline$\hat{\bar{Y}}_{15(s y)}$ & 146.004 & 22523.33 & 167.4054 \\
\hline$\hat{\bar{Y}}_{16(s y)}$ & 157.1975 & 17099.3 & 220.5076 \\
\hline$\hat{\bar{Y}}_{17(s y)}$ & 1.21033 & 36629.17 & 524.2826 \\
\hline$\hat{\bar{Y}}_{18 p(s y)}$ & 73.873 & 7321.673 & 575.1886 \\
\hline$\hat{\bar{Y}}_{19 p(s y)}$ & 73.0208 & 7191.781 & 210.4772 \\
\hline$\hat{\bar{Y}}_{20 p(s y)}$ & 68.10311 & 6555.287 & \\
\hline$\hat{\bar{Y}}_{21 p(s y)}$ & 2.106374 & 138.7300 & 4834.807 \\
\hline$\hat{\bar{Y}}_{22 p(s y)}$ & 77.83677 & & \\
\hline & & & \\
\hline
\end{tabular}


From Table 1, it can be seen that proposed estimators $\left(\hat{\bar{Y}}_{18 p(s y)}, \hat{\bar{Y}}_{19 p(s y)}, \hat{\bar{Y}}_{20 p(s y)}\right.$ and $\left.\hat{\bar{Y}}_{21 p(s y)}\right)$ have greater relative efficiency than systematic version of Suberamani and Kumarpandiyan (2013) estimator $\left(\hat{\bar{Y}}_{6(s y)}\right)$, Yan and Tian (2010) estimator $\left(\hat{\bar{Y}}_{3(s y)}\right)$, Singh et al (2004) estimator $\left(\hat{\bar{Y}}_{2(s y)}\right)$, Kadilar and Cigni (2004) estimator $\left(\hat{\bar{Y}}_{8(s y)}\right)$ respectively. We can also observed that proposed estimators $\left(\hat{\bar{Y}}_{18 p(s y)}, \hat{\bar{Y}}_{19 p(s y)}, \hat{\bar{Y}}_{20 p(s y)}\right.$ and $\left.\hat{\bar{Y}}_{21 p(s y)}\right)$ have smaller biases than $\hat{\bar{Y}}_{6(s y)}, \hat{\bar{Y}}_{3(s y)}, \hat{\bar{Y}}_{2(s y)}$ and $\hat{\bar{Y}}_{8(s y)}$ respectively. Hence, it is clear that the proposed estimators in systematic sampling are more efficient and have smaller bias than corresponding parent estimators (estimator from which they are derived). Furthermore, the proposed ratio -cum- product type estimator $\left(\hat{\bar{Y}}_{22 p(s y)}\right)$ that utilize a linear combination of mean and median of two auxiliary variables in systematic sampling are more efficient than systematic version of Suberamani and Kumarpandiyan (2013) estimator ( $\left.\hat{\bar{Y}}_{6(s y)}\right)$ and Tailor et al. (2013) estimator $\left(\hat{\bar{Y}}_{17(s y)}\right)$ that utilize median of only one auxiliary variable. It can also be inferred from the table that the proposed estimators $\left(\hat{\bar{Y}}_{20 p(s y)}\right)$ and $\left(\hat{\bar{Y}}_{22 p(s y)}\right.$ ) have a highest percentage relative efficiency, i.e. 575.1886 and 779.8711 , as compare to other estimators discussed in this chapter. At same time, we can also observed that proposed estimator $\left(\hat{\bar{Y}}_{22 p(s y)}\right)$ that utilize a linear combination of mean and median of two auxiliary variables in systematic sampling are less bias than Suberamani and Kumarpandiyan (2013) estimator $\left(\hat{\bar{Y}}_{6(s y)}\right)$ that utilize median of only one auxiliary variable. So it can be inferred from table the proposed estimator $\hat{\bar{Y}}_{21 p(s y)}$ have a less bias 2.106374 as compare to other estimator discuss in this chapter.

\section{Conclusion}

The paper covered utilization of different known parameters of auxiliary variable (i.e. mean, median, coefficient of variation, coefficient of kurtosis and skewness etc) in constructing estimators for population mean under systematic sampling. We also proposed some new ratio and product type estimator, for using median along with mean of two auxiliary variables in systematic random sampling to improve efficiency. These proposed estimators perform better than existing estimator in the term of percent relative efficiency. So we recommend to use these estimators for future research when these information are easily obtainable.

\section{Acknowledgement}

Authors are thankful to the editorial team for their support and guidance.

\section{References}

Bahl, S. and Tuteja, R.K. (1991). Ratio and product type exponential estimator. Journal of Infrm. and Optim. Sci., 12.(1), 159-163. 
Chaudhary, M. A. and Kumar, A. (2015). Estimating the Population Mean Using Two-Phase Sampling in the Presence of Non-Response. Journal of World Applied Sciences, 33.(6), 874-882.

Cochran, W. G. (1940). The estimation of the yields of cereal experiments by sampling for the ratio of grain to total produce. The Journal of Agricultural Science, 30. (2), 262-275.

Gupta, S. and Shabbir, J. (2011). On estimating the finite population mean in simple and Stratified random sampling. Journal of Communication in Statistics.

Kadilar, C. and Cingi, H. (2004). Ratio estimators in simple random sampling. Journal of Applied Mathematics and Computation, 151, 893-902.

Kadilar, C. and Cingi, H. (2006). An Improvement in Estimating the Population mean by using the Correlation Coefficient. Journal of Mathematics and Statistics, 35. (1), 103109.

Khan, M. and Singh, R. (2015). Estimation of Population Mean in Chain Ratio-Type Estimator under Systematic Sampling. Journal of Probability and Statistics, 1, 1-5.

Khan, M., Ullah, S., Al-Hossain, A. Y. and Bashir, N. (2015). Improved ratio-type estimators using maximum and minimum values under simple random sampling scheme. Journal of Mathematics and Statistics, 44. (4), 923-93.

Singh, H.P., Tailor, R., Tailor, R. and Kakran, M.S. (2004). An Improved Estimator of population mean using Power transformation, Journal of the Indian Society of Agricultural Statistics 58(2), 223-230.

Singh, H. P., Tailor, R. and Jatwa, N. K. (2011). Modified ratio and product estimators for Population Mean in Systematic Sampling. Journal of Modern Applied Statistical Methods, 10. (2), 424-435.

Singh, R. and Singh, H. P. (1998). Almost unbiased ratio and product-type estimators in systematic sampling. 22. (3), 403-416.,

Singh, R., Malik, S. and Singh, V.K. (2012). An improved estimator in systematic sampling, Journal of Scientific Research, 56, 177-182.

Sisodia, B. V. S. and Dwivedi, V. K., (1981). A Modified Ratio Estimator Using Coefficient of Variation of Auxiliary Variable. Journal of Indian Society Agricultural Statistics, 33, 1318. 
Subramani, J. and Kumarapandiyan, G. (2013). New Modified Ratio Estimator for Estimation of Population Mean when Median of the Auxiliary Variable is known, 9.(2), 137-145.

Subramanian, J. and Kumarapandiyan, G. (2012). Estimation of Population Mean Using Known Median and Co-Efficient of Skewers. American Journal of Mathematics and Statistics, 2. (5), 101-107.

Singh, M. P., (1967). Ratio-cum-product method of estimation. 12, 34-42.

Tailor, R., Jatwa, K. and Singh, P. (2013). A ratio-cum-product estimator of finite population mean in systematic sampling. Journal of statistics in transition new series, 14(3). 391398.

Upadhyaya, L. N. and Singh, H. P. (1999). Use of Transformed Auxiliary Variable in Estimating the Finite Population Mean. Journal of Biometrical 41. ( 5) 627-636.

Yan, Z. and Tian, B. (2010). Ratio Method to the Mean Estimation Using Coefficient of Skewness of Auxiliary Variable, 106, 103-110.

Data Source 1: District wise maize data of Punjab, (2014). URL: http://www.amis.pk/ Agristatistics/ DistrictWise/ Maize.html 1/6) 